\title{
Orchid diversity in three districts [Kiphire, Tuensang and Zunheboto] of Nagaland, India
}

\author{
Chitta Ranjan Deb ${ }^{1}$ and Hutuka Y. Jakha \\ Department of Botany, Nagaland University, Lumami 798627, Nagaland, India \\ ${ }^{1}$ Communicating author, e-mail: debchitta@rediffmail.com and debchitta@gmail.com
}

[Received 13.11.2019; Revised 10.12.2019; Accepted 11.12.2019; Published 31.12.2019]

\begin{abstract}
Orchids in India are mostly confined to North-Eastern region and Western Ghats. Nagaland, a state of NE India is a hilly state with varied agro-climatic condition supports large number of orchids in different habitats. This study was an attempt for inventory of wild orchid resources of Kiphire, Tuensang and Zunheboto districts of Nagaland. Outcome of the study is the checklist of orchids diversity based on only primary data from the present study. A total of 180 species from 58 genera are collected, studied, plants are being maintained in the Orchidarium in the Department of Botany, Nagaland University. Of the, 180 species, 30 are epiphytic, 15 terrestrial, 11 are of dual habitat (terrestrial/epiphytic) and 2 are saprophytes.
\end{abstract}

Key words: Nagaland districts, Kiphire, Tuensang, Zunheboto, Orchid diversity

\section{INTRODUCTION}

Orchidaceae are one of the largest families of flowering plants accounting $~ 7 \%$ of the total flowering plants. Orchids are known for their beautiful flowers of different sizes, fascinating shapes and beautiful colors which have outnumbered other families of flowering plants by evolving higher levels of specialization in its vegetative and reproductive traits. World estimate of orchid species has been usually assessed between 17,000 and 35,000 (Dressler 1993). Some of the conservative estimates on this family, however, suggest 19,128 species in 800 genera (Atwood 1986); 18,000 species in 750 genera (Haywood 1993); 18,500 species in 778 genera (Mabberley 1998) and20,000 species by Lawler \& Rao (2002). Recent estimates suggests approximately 26,567 species are distributed worldwide(WCSP 2017). Unfortunately, orchids have become victims of their own beauty and utility and their natural populations have been detrimentally affected by unscrupulous collections for commercial and scientific purposes. In 1975, the Convention on International Trade in Endangered Species of Wild Fauna and Flora (CITES), suggested a complete ban on the export of wild orchid plants to avoid overutilization. Orchids have been used in different systems of local medicine since the Vedic period (Deb et al. 2009). Besides this, orchids also find their use as an important ornamental crop in floriculture industry due to their beautiful foliage (Deb \& Imchen 2011).

The first account on the work of 'Orchidaceae of Nagaland' was Clarke's (1889) collection wherein reported 22 species under 18 genera followed by Hooker (1890) recorded 49 species under 24 genera. Recent works on the Orchidaceae of Nagaland includes Chankija et al. (1992) recorded 360 species under 85 genera, Deorani \& Naithani (1995) reported 238 species, Hynniewta et al. (2000) recorded 241 species of 63 genera, Deb \& Imchen (2008) recorded 396 species of 92 genera. Post 2008 studies added several species to the 
orchid flora of Nagaland viz., Dendrobium tuensangense (Odyuo et al. 2017); Arachnis labrosa var. zhaoi (Jakha et al. 2015a), Coelogyne calcicola (Odyuo et al. 2016a), Panisea panchassensis (Odyuo et al.2016b), Dendrobium regium(Deb et al. 2015), Polystachya concreta (Deb et al. 2016), Esmeralda clarkei (Deb et al, 2014), Dendrobium tamenglongense (Jakha et al. 2014a), Arachnis senapatiana (Jakha et al. 2014b), Pendulorchis himalaica (Jakha et al. 2015b), Goodyera fumata; Phalaenopsis manii; Vanilla parishii (Jamir et al. 2015), Phalaenopsis braceana (Imchen et al. 2015), Crepidium calophyllum; Eriodes barbata; Erythrodes blumei (Jakha \& Dey 2017), Cymbidium bicolor (Deb et al. 2017) etc. Present study was undertaken to survey and documentation of orchid resources of three districts of Nagaland viz., Kiphire, Tuensang and Zunheboto.

\section{MATERIALS AND METHODS}

\section{Study Area}

The three districts namely Kiphire, Tuensang and Zunhebeto are predominantly mountainous, altitudinal variations ranging from approximately $300 \mathrm{~m}$ in the lower regions of the Zunheboto side of Doyang River to $3840 \mathrm{~m}$ Mt. Saramati, in Pungro sub-division of Kiphire district and diverse species of orchids are predominant across these altitudinal ranges.

Kiphire district: Kiphire district is surrounded by Tuensang district in the North, Phek district in the South, Zunheboto in the West and Myanmar in the East occupying 1255 sq $\mathrm{km}$. The district headquarters Kiphire is located at an elevation of $896.42 \mathrm{~m}$ MSL. The climate is usually humid and hot during summer and cold during winter with temperature range between $2.7^{\circ} \mathrm{C}$ during winter and a high of $37^{\circ} \mathrm{C}$ during summer. Monsoon period starts from June up to September with $876 \mathrm{~mm}$ average rain fall.

Tuensang district: Tuensang district is located in the eastern part of Nagaland and surrounded by Mon and Longleng districts in the North, Mokokchung in the North-West, Zunheboto in the South-West, Kiphire in the South, and Myanmar in the East. The district has an area of $\sim 1,728 \mathrm{sq} \mathrm{km}$. The climate of the district ranges from sub-tropical to temperate.

Zunheboto district: Zunheboto district is bordered on the North by Mokokchung district, Kohima district and Wokha district on the West, Phek district in the South, and Tuensang district and Kiphire district in the East. The district has an area of about 1, $255 \mathrm{sq} \mathrm{km}$.

Extensive field survey was carried out at various village forest areas of Kiphire, Tuensang and Zunheboto districts (Table 1) during 2013 - 2017in different seasons of the year to gather comprehensive information on the diversity of orchid species. Since the forest is mostly owned by the community, due permission from the village 'Gaonbura/Chairman' were obtained to enter forest areas of respective village. The specimens have been collected with GPS data and field photographs.

\section{Method of collection and herbarium preparation}

Random collection in village forest areas were carried out referring the forest cover map of the three districts viz., Kiphire, Tuensang and Zunheboto. Efforts were made to visit the more dense forest areas which were relatively undisturbed. Photographs of plants in flowering were taken along with GPS coordinates. The specimens collected from the field are accorded with proper field numbers and then pressed, dried and made into herbarium sheets as per standard method (Jain \& Rao 1977). Fragile parts of plants such as flowers were also preserved and pickled in formaldehyde for later dissection. All herbarium sheets have been deposited in the herbarium of Department of Botany, Nagaland University, Lumami. 


\section{Live specimen and identification}

Live specimens were brought and maintained in the Orchidarium of Department of Botany, Nagaland University for further observation of flowering and other characteristics. Identification of plants was done referring the regional and national flora sand also with the help of relevant taxonomic revisions, monographs etc. Later, the identities of the species were confirmed by consulting the herbaria of Botanical Survey of India, Eastern Regional Centre, Shillong (ASSAM) and Central National Herbarium, Botanical survey of India, Howrah (CAL).

\section{RESULTS AND DISCUSSION}

The present floristic study documents a total of 180 species under 58 genera (Table 2). The genera Dendrobium, Bulbophyllum and Coelogyne are having 29, 17 and 15 species respectively and other genera are represented by lesser number of species (Table 3). Out of the 58 genera, 30 genera were strictly epiphytic, while 15 genera were terrestrial and 11 genera belonging to the mixed type i.e. either terrestrial or epiphytic. Epipogium and Eulophia species were found to be saprophytic and was represented by 1 species each, namely Epipogium roseum and Eulophia zollengeri. The species viz. Polystachya concreta, Esmeralda clarkei, Dendrobium tamenglongense, Arachnis senapatiana, Phalaenopsis braceana, Cymbidium bicolor, Erythrodes blumei, Eriodes barbata, Crepidium calophyllum, Pendulorchis himalaica for the state, and Arachnis labrosa var. zhaoi and Thrixspermum laurisilvaticum for the country are already newly reported by the present authors (Deb et al. 2014, 2015, 2016, 2017). Reports of Dendrobium tuensangense N. Odyuo \& C. Deori, a new species from Tuensang district in 2016 and Panisea panchaseensis Subedi also from Tuensang district a new record for the country by Odyuo et al. in 2016 have not been included in the list since the authors have not come across the specimens.

From the field studies it is observed that the species of the genera Bulbophyllum, Coelogyne, Cymbidium, Dendrobium, Eria, Goodyera, Papilionanthe, Pinalia, Thrixspermum, Vanda are growing in wide altitudinal range, while species of the genera Epigenium, Phalaenopsis, Panisea, Pleione, Renanthera, Vandopsis, are found to occur only at above $2000 \mathrm{~m}$ altitude.

In the study areas different types of vegetation observed and different vegetation supported the growth of different orchid species as follows:

Tropical vegetation: Among the three districts, only a small patch on the Zunheboto side of Doyang River could be classified under this type of vegetation and is a home to a wide variety of orchid species of both epiphytic and terrestrial. Epiphytic species viz. Acampe papillosa, Aerides odorata, Bulbophyllum caryanum, Cleisostoma paniculatum, $C$. williamsonii, Coelogyne fimbriata, C. ovalis, Cymbidium aloifolium, C. iridiodes, Dendrobium aphyllum, D. chrysotoxum, Luisia trichorrhiza, Papilionanthe teres, Pinalia amica, Polystachya concreta, Vanda testaceae and terrestrial orchids viz. Calanthe triplicata, Crepidium acuminatum, C. calophyllum, Dienia ophrydis, Phaius tankervilleae, Tainia latifolia are predominant.

Sub-Tropical vegetation: Majority of the orchid species were found to occur in this vegetation type viz., Acampe ochracea, A. rigida, Aerides multiflora, A. odorata, Agrostophyllum callosum, Arachnis labrosa, A. labrosa var zhaoi, Bulbophyllum affine, B. caryanum, B. cariniflorum, B. crassipes, B. cylindraceum, B. leopardinum, B. pteroglossum, B. rotschildianum, Cleisocentron pallens, Cleisostoma parishii, 
206 Orchid diversity in three districts of Nagaland, India

Table 1. G.P.S. points of the areas and localities of the three districts visited during field survey

\begin{tabular}{|c|c|c|c|c|}
\hline $\begin{array}{l}\text { Sl. } \\
\text { No }\end{array}$ & Area & District & $\begin{array}{l}\text { Position with approx. } \\
\text { altitude }\end{array}$ & Localities visited (villages) \\
\hline 1. & Pungro & Kiphire & $\begin{array}{l}25^{\circ} 50^{\prime} 07.1^{\prime \prime} \mathrm{N} \mathrm{\&} \\
94^{\circ} 51^{\prime} 25.2^{\prime \prime} \mathrm{E}, 1343 \mathrm{~m}\end{array}$ & $\begin{array}{l}\text { Chomi, Iponger, khongkha, Moya, } \\
\text { Mimi, Fakim }\end{array}$ \\
\hline 2. & $\begin{array}{l}\text { Khongsa } \\
\text { (Saramati) }\end{array}$ & Kiphire & $\begin{array}{l}25^{\circ} 45^{\prime} 13 ” \mathrm{~N} \mathrm{\&} \\
94^{\circ} 59 ' 24.5 ’ \mathrm{E}, 2705 \mathrm{~m}\end{array}$ & $\begin{array}{l}\text { Khong, Penkim, Salomi, } \\
\text { Thanamer, Sangtsung }\end{array}$ \\
\hline 3. & Longmatra & Kiphire & $\begin{array}{l}25^{\circ} 50^{\circ} 08^{\prime \prime} \mathrm{N} \& \\
94^{\circ} 51^{\prime} 25.6 ” \mathrm{E}, 1343 \mathrm{~m}\end{array}$ & $\begin{array}{l}\text { Ngoromi, Sangphure, Sangtsoze, } \\
\text { Tethuze }\end{array}$ \\
\hline 4. & Seyochung & Kiphire & $\begin{array}{l}25^{\circ} 53^{\prime} 175^{\prime \prime} \text { N \& } \\
094^{\circ} 38^{\prime} 327^{\prime} \text { ' E, } 2041 \mathrm{~m}\end{array}$ & $\begin{array}{l}\text { Lukhami, Yangzitong, Yingphire, } \\
\text { Thsinger }\end{array}$ \\
\hline 5. & Sitimi & Kiphire & $\begin{array}{l}25^{\circ} 47^{\prime} 18.6 ” \mathrm{~N} \& \\
94^{\circ} 44^{\prime} 44.77^{\prime} \mathrm{E}, 1282 \mathrm{~m}\end{array}$ & $\begin{array}{l}\text { Honito, Kiyezhe, Thazuvi, Nikiye, } \\
\text { Nitoi, Shishimi, Xuvishe }\end{array}$ \\
\hline 6. & Amahotor & Kiphire & $\begin{array}{l}25^{\circ} 56^{\prime} 621^{\prime \prime} \mathrm{N} \& 094^{\circ} \& \\
44^{\prime} 659^{\prime \prime} \mathrm{E}, 1976 \mathrm{~m}\end{array}$ & Hutanger, hurong, kisetong, Purur \\
\hline 7. & Longkhim & Tuensang & $\begin{array}{l}26^{\circ} 12 \text { '24.3”N \& } \\
94^{\circ} 39^{\prime} 09.7 ’ \mathrm{E}, 1585 \mathrm{~m}\end{array}$ & $\begin{array}{l}\text { Angangba, Chimonger, Mangakhi, } \\
\text { New Sangsamong, Sangsamong }\end{array}$ \\
\hline 8. & Sotokur & Tuensang & $\begin{array}{l}26^{\circ} 12 \text { '11.1”N \& } \\
94^{\circ} 44^{\prime} 44.3 ” \mathrm{~B}, 2200 \mathrm{~m}\end{array}$ & Helipong, Sipongsang, Kuthur \\
\hline 9. & Chare & Tuensang & $\begin{array}{l}26^{\circ} 16^{\prime} 20.7 ’ \mathrm{~N} \mathrm{\&} \\
94^{\circ} 36^{\prime} 04.3 ’ \mathrm{E}, 959 \mathrm{~m}\end{array}$ & $\begin{array}{l}\text { Alisophur, Chare village, } \\
\text { Thronger, Tsadang (Old and } \\
\text { New), Kiding }\end{array}$ \\
\hline 10. & Chingmei & Tuensang & $\begin{array}{l}26^{\circ} 14^{\prime} 00.4^{\prime \prime} \mathrm{N} \& \\
94^{\circ} 56^{\prime} 39.2^{\prime \prime} \mathrm{E}, 1438 \mathrm{~m}\end{array}$ & $\begin{array}{l}\text { Chendang saddle village, Waoshu, } \\
\text { Chingmei village, Yimpang }\end{array}$ \\
\hline 11. & Noklak & Tuensang & $\begin{array}{l}26^{\circ} 15^{\prime} 26.8^{\prime \prime} \mathrm{N} \& \\
95^{\circ} 06 ' 59.3 ” \mathrm{E}, 1969 \mathrm{~m}\end{array}$ & $\begin{array}{l}\text { Dan village, Pangsha (Old and } \\
\text { New), Wansoi, Nokyan, Noklak }\end{array}$ \\
\hline 12. & Shamator & Tuensang & $\begin{array}{l}26^{\circ} 06 ’ 07.6 ” \mathrm{~N} \& \\
94^{\circ} 50 \text { '23.1'E, } 2153 \mathrm{~m}\end{array}$ & $\begin{array}{l}\text { Meliankiur, Shiponger, } \\
\text { Waphur,Yakor }\end{array}$ \\
\hline 13. & Satoi & Zunheboto & $\begin{array}{l}25^{\circ} 49^{\prime}, 10.3^{\prime} \mathrm{N} \mathrm{\&} \\
94^{\circ} 35^{\prime} 42.3 \mathrm{E}, 2324 \mathrm{~m}\end{array}$ & $\begin{array}{l}\text { Hokiye, Khuvuxu, Satoi village, } \\
\text { Tsutoho }\end{array}$ \\
\hline 14. & Ghathashi & Zunheboto & $\begin{array}{l}25^{\circ} 39 \text { '25.3”N \& } \\
94^{\circ} 21^{\prime} 35.3 \mathrm{E}, 432 \mathrm{~m} ; \\
25^{\circ} 53 \text { '18.3” N \& } \\
94^{\circ} 22^{\prime} 44.0^{\prime \prime} \text { E. } 886 \mathrm{~m}\end{array}$ & $\begin{array}{l}\text { Mukalimi, Chishilimi, Chisholimi, } \\
\text { Khughutomi, Kilomi }\end{array}$ \\
\hline 15. & Atoizu & Zunheboto & $\begin{array}{l}26^{\circ} 06^{\prime} 31.6 ” \mathrm{~N} \& \\
94^{\circ} 30^{\prime} 56.4 \text { " E. } 1806 \mathrm{~m}\end{array}$ & $\begin{array}{l}\text { Naghuto (Old and New), Atoizu } \\
\text { village, Litsami, Emlomi }\end{array}$ \\
\hline 16. & Pughoboto & Zunheboto & $\begin{array}{l}25^{\circ} 51^{\prime} 12.0^{\prime \prime} \mathrm{N} \& \\
94^{\circ} 19^{\prime} 19.4^{\prime \prime} \mathrm{E}, 835 \mathrm{~m}\end{array}$ & $\begin{array}{l}\text { Mishilimi, Lazami, Natsumi, } \\
\text { Ghokimi, Tsaphimi }\end{array}$ \\
\hline 17. & $\begin{array}{l}\text { V.K. Area } \\
\text { village }\end{array}$ & Zunheboto & $\begin{array}{l}26^{\circ} 06^{\prime} 47.7 ” \mathrm{~N} \& \\
94^{\circ} 23 \text { '24.8”' E. } 401 \mathrm{~m}\end{array}$ & Mukhami, Izheto \\
\hline 18. & Akuluto & Zunheboto & $\begin{array}{l}26^{\circ} 12 \text { '11.4”' N \& } \\
94^{\circ} 29^{\prime} 21.0 \text { "'E.971 m }\end{array}$ & $\begin{array}{l}\text { Lumami, Alaphumi, Sema settsu, } \\
\text { Sutemi, Zaphumi, Lumithsami }\end{array}$ \\
\hline 19. & Saptiqa & Zunheboto & $\begin{array}{l}25^{\circ} 58 \text { '40”N \& } \\
94^{\circ} 24^{\prime} 22.2 \text { 'E. } 901 \mathrm{~m}\end{array}$ & Ustomi, \\
\hline 20. & $\begin{array}{l}\text { Zunheboto } \\
\text { sadar }\end{array}$ & Zunheboto & $\begin{array}{l}26^{\circ} 05 \text { '27.3” N \& } \\
94^{\circ} 32 \text { '46.9”'E. } 1936 \mathrm{~m}\end{array}$ & Asukhomi, Sukhalu, Lochomi \\
\hline 21. & Aghunato & Zunheboto & $\begin{array}{l}25^{\circ} 57^{\prime} 9.1^{\prime \prime} \mathrm{N} \& \\
94^{\circ} 36^{\prime} 12.7^{\prime \prime} \mathrm{E}, 1314 \mathrm{~m}\end{array}$ & $\begin{array}{l}\text { Aquba (Old and New), Lukhuyi, } \\
\text { Thokihi }\end{array}$ \\
\hline 22. & Satakha & Zunheboto & $\begin{array}{l}25^{\circ} 57 \text { '58.9” N \& } \\
94^{\circ} 24^{\prime} 25.5^{\prime \prime} \mathrm{E}, .920 \mathrm{~m}\end{array}$ & $\begin{array}{l}\text { Nunumi, Zhekiye, Khukiye, } \\
\text { Sukhai, Shoipu }\end{array}$ \\
\hline
\end{tabular}


C. williamsonii, Coelogyne cristata, C. fimbriata, C. ovalis, C. prolifera, C. schultesii, C. Suaveolens, Cryptochilus luteus, C. sanguineus, Cymbidium aloifolium, C. bicolor, C. iridiodes, D. aphyllum, D. chrysanthum, D. chrysotoxum, D. densiflorum, D. formosum, D. jenkinsii, D. moschatum, D. ochreatum, D. spatella, D. transparens, Epigeneium amplum, Eria spicata, Gastrochilus calceolaris, $G$. inconspicua, Liparis bootanensis, L. distans, L. viridiflora, Luisia trichorrhiza, Oberonia pachyrachis, Papilionanth vandarum, Phalanaeopsis difformis, Pholidota articulate, Pinalia acervata, P. paniculata, P. pannea, Pleione maculata, Polystachya concreta, Vanda ampullaceaum, V. bicolor, V. coerulea, V. testaceae and terrestrial orchids such as Acanthephippium striatum, Anthogonium gracile, Arundina graminifolia, Calanthe biloba, C. mannii, Calanthe puberula, C.triplicata,

Table 2. List of orchid species documented from the three districts viz. Kiphire, Tuensang and Zunheboto. [Total: 180 species belonging to 58 Genera]

\begin{tabular}{|c|c|c|c|}
\hline Name of Genera & & Name of Species & Flowering \\
\hline Acampe & 1 & Acampe praemorsa(Roxb.) Blatt. \& McCann & Dec-Jan \\
\hline & 2 & Acampe ochracea (Lindl.) Hochr. & Jan - Feb \\
\hline & 3 & Acampe rigida (Buch.-Ham. ex Sm.) P.F.Hunt & Aug - Oct \\
\hline Acanthephippium & 4 & Acanthephippium striatum Lindl. & May - Jun \\
\hline Aerides & 5 & Aerides multiflora Roxb. & Apr-May \\
\hline & 6 & Aerides odorata Lour. & May - Jun \\
\hline Agrostophyllum & 7 & Agrostophyllumcallosum Rchb.f. & Jun - Jul \\
\hline Anthogonium & 8 & Anthogonium gracile Lindl. & Aug - Sep \\
\hline Arachnis & 9 & Arachnis labrosa (Lindl. ex Paxt) Rchb. f. & Aug - Sep \\
\hline & 10 & Arachnis labrosa var Zhaoi & Aug - Sep \\
\hline & 11 & $\begin{array}{l}\text { Arachnis senapatiana(Phukan \& A.A.Mao) Kocyan \& } \\
\text { Schuit. }\end{array}$ & May - Jun \\
\hline Arundina & 12 & Arundina graminifolia (D. Don) Hochr. & Jun - Aug \\
\hline Bulbophyllum & 13 & Bulbophyllum affine Lindl. & May - Jun \\
\hline & 14 & Bulbophyllum candidum Hook.f. & Aug - Sep \\
\hline & 15 & Bulbophyllum caryanum(Hook.) Spreng & June - Jul \\
\hline & 16 & Bulbophyllum cariniflorum Rchb.f. & Mar-Apr \\
\hline & 17 & Bulbophyllum crassipes Hook.f. & Sep-Nov \\
\hline & 18 & Bulbophyllum cylindraceum Lindl. & Sep - Oct \\
\hline & 19 & Bulbophyllum elatum (Hook.f.) J.J.Sm. & May - Jun \\
\hline & 20 & Bulbophyllum. helenae ( Kuntze.) J.J.Sm. & May - Jun \\
\hline & 21 & Bulbophyllum leopardinum (Wall.) Lindl. & Jun - Jul \\
\hline & 22 & Bulbophyllum odorotissimum (Sm.) Lindl. & May - Jun \\
\hline & 23 & Bulbophyllum pteroglossum Schltr. & Oct - Nov \\
\hline & 24 & Bulbophyllum reptans Lindl. & Oct - Nov \\
\hline & 25 & Bulbophyllum retusiusculum Rchb. & Aug - Sep \\
\hline & 26 & Bulbophyllum rotschildianum (O’Brien) J.J. Sm. & Sep - Oct \\
\hline & 27 & Bulbophyllum sunipia J.J.Verm., Schuit. \& de Vogel & May - Jun \\
\hline & 28 & Bulbophyllum umbellatum Lindl., & Apr - Jun \\
\hline & 29 & Bulbophyllum viridiflorum (Hook.f.) Schltr. & Oct - Nov \\
\hline Bulleyia & 30 & Bulleyia yunnanensis Schltr. & Jun - Jul \\
\hline Calanthe & 31 & Calanthe alismifolia Lindl. & Jun - Jul \\
\hline & 32 & Calanthe biloba Lindl. & Nov - Dec \\
\hline
\end{tabular}


208 Orchid diversity in three districts of Nagaland, India

\begin{tabular}{|c|c|c|c|}
\hline \multirow{2}{*}{$\begin{array}{l}\text { Name of Genera } \\
\text { Calanthe (contd.) }\end{array}$} & \multicolumn{2}{|r|}{ Name of Species } & \multirow{2}{*}{$\begin{array}{c}\begin{array}{c}\text { Flowering } \\
\text { Season }\end{array} \\
\text { May - Jun } \\
\end{array}$} \\
\hline & 33 & Calanthe brevicornu Lindl. & \\
\hline & 34 & Calanthe mannii Hook.f. & Apr - May \\
\hline & 35 & Calanthe puberula Lindl. & May - Jun \\
\hline & 36 & Calanthe triplicate (Willem.) Ames. & Jul-Oct \\
\hline Chiloschista & 37 & Chiloschista parishii Seidenf. & Apr - May \\
\hline Cleisocentron & 38 & $\begin{array}{l}\text { Cleisocentron pallens(Cathcart ex Lindl.) N. Pearce \& } \\
\text { P.J.Cribb }\end{array}$ & Sep-Oct \\
\hline \multirow[t]{3}{*}{ Cleisostoma } & 39 & Cleisostoma paniculatum (Ker Gawler) Garay. & Jul - Aug \\
\hline & 40 & Cleisostoma parishii (Hook.f.) Garay & Sep \\
\hline & 41 & Cleisostoma williamsonii (Reichb.f.) Garay & Jun - Jul \\
\hline \multirow[t]{15}{*}{ Coelogyne } & 42 & Coelogyne barbata Lindl. ex Griff. & Oct - Dec \\
\hline & 43 & Coelogyne calcicola Kerr. & May - Jun \\
\hline & 44 & Coelogyne corymbosa Lindl. & Mar-Apr \\
\hline & 45 & Coelogyne cristata Lindl. & Mar-May \\
\hline & 46 & Coelogyne fimbriata Lindl. & Oct - Dec \\
\hline & 47 & Coelogyne flaccida Lindl. & Mar \\
\hline & 48 & Coelogyne griffithii Hook.f. & Mar - May \\
\hline & 49 & Coelogyne nitida (Wall. ex D. Don) Lindl. & June-July \\
\hline & 50 & Coelogyne occultata Hook.f. & Jun - Jul \\
\hline & 51 & Coelogyne ovalis Lindl. & Sept - Oct \\
\hline & 52 & Coelogyne prolifera Lindl. & May - Jun \\
\hline & 53 & Coelogyne punctulata Lindl. & Mar-Apr \\
\hline & 54 & Coelogyne schultesii S.K.Gen \& S.Das & Mar \\
\hline & 55 & Coelogyne stricta(D. Don) Schltr. & Apr-May \\
\hline & 56 & Coelogyne. suaveolens Hook.f. & May - Jun \\
\hline Cremastra & 57 & Cremastra appendiculata (D.Don) Makino & May - Jun \\
\hline \multirow[t]{3}{*}{ Crepidium } & 58 & Crepidium acuminatum (D.Don) Szlach. & Jun - Jul \\
\hline & 59 & Crepidium calophyllum (Rchb.f.) Szlach. & Jun - Jul \\
\hline & 60 & Crepidium khasianum (Hook.f.) Szlach. & Jun - Jul \\
\hline \multirow[t]{2}{*}{ Cryptochilus } & 61 & Cryptochilus luteus Lindl. & May - Jun \\
\hline & 62 & Cryptochilus sanguineus Wall. & Jun - Jul \\
\hline \multirow[t]{9}{*}{ Cymbidium } & 63 & Cymbidium aloifolium(L.) Sw. & Apr - May \\
\hline & 64 & Cymbidium bicolor Lindl. & Mar-Apr \\
\hline & 65 & Cymbidium cochleare Lindl. & Nov - Dec \\
\hline & 66 & Cymbidium devonianum Paxton & Apr - May \\
\hline & 67 & Cymbidium elegans Lindl. & Nov - Dec \\
\hline & 68 & Cymbidium erythraeum Lindl. & Nov - Dec \\
\hline & 69 & Cymbidium iridiodes D. Don & Oct - Jan \\
\hline & 70 & Cymbidium mastersii Griff. ex Lindl. & Oct - Nov \\
\hline & 71 & Cymbidium tigrinum Par. ex Hook & Apr - May \\
\hline \multirow{9}{*}{ Dendrobium } & 72 & Dendrobium aphyllum (Roxb.) Fischer & Apr - May \\
\hline & 73 & Dendrobium chrysanthum Wall. exLindl. & Sep-Oct \\
\hline & 74 & Dendrobium chrysotoxum Lindl. & Apr - May \\
\hline & 75 & Dendrobium crepidatum Lindl. \& Paxt & Apr - May \\
\hline & 76 & Dendrobium densiflorum (Lindl.) Wall. & Apr - May \\
\hline & 77 & Dendrobium denudans D.Don & Nov - Dec \\
\hline & 78 & Dendrobium devonianum Paxt. & May - Jun \\
\hline & 79 & Dendrobium falconeri Hook.f. & Apr - May \\
\hline & 80 & Dendrobium fimbriatum var.oculatum Hook.f. & Apr-May \\
\hline
\end{tabular}


Chitta Ranjan Deb \& Hutuka Y. Jakha 209

\begin{tabular}{|c|c|c|c|}
\hline \multirow{3}{*}{$\begin{array}{l}\text { Name of Genera } \\
\begin{array}{l}\text { Dendrobium } \\
\text { (contd.) }\end{array}\end{array}$} & \multicolumn{2}{|r|}{ Name of Species } & \multirow{2}{*}{$\begin{array}{c}\begin{array}{c}\text { Flowering } \\
\text { Season }\end{array} \\
\text { Jun }- \text { Jul } \\
\end{array}$} \\
\hline & 81 & Dendrobium formosumRoxb. Ex Lindl. & \\
\hline & 82 & Dendrobium gibsonii Lindl. & Jun - Jul \\
\hline & 83 & Dendrobium heterocarpum Wall. ex Lindl. & Mar-Apr \\
\hline & 84 & Dendrobium jenkinsii Wall. ex Lindl. & Mar - Apr \\
\hline & 85 & Dendrobium lituiflorum Lindl. & Mar - Apr \\
\hline & 86 & Dendrobium longicornu Lindl. & Oct - Nov \\
\hline & 87 & Dendrobium moschatum Griff. & Jun - Jul \\
\hline & 88 & Dendrobium nobile Lindl. & Apr - May \\
\hline & 89 & Dendrobium ochreatum Wall. ex Lindl. & Apr - May \\
\hline & 90 & Dendrobium parishii Rchb.f. & Apr - May \\
\hline & 91 & Dendrobium polyanthum Wall. ex Lindl. & Mar - Apr \\
\hline & 92 & Dendrobium porphyrochilum Lindl. & May - Jun \\
\hline & 93 & Dendrobium sociale J.J.Sm. & Mar - Apr \\
\hline & 94 & Dendrobium spatella Rchb.f. & Jun - Aug \\
\hline & 95 & $\begin{array}{l}\text { Dendrobium tamenlongse R. Kishor; Y.N. Devi; H.B. } \\
\text { Sharma; J. Tongbram \& S.P. Vij }\end{array}$ & Jul - Aug \\
\hline & 96 & Dendrobium thyrsiflorum Rchb. f. & Sep - Oct \\
\hline & 97 & Dendrobium transparens Wall. ex Lindl. & Apr - May \\
\hline & 98 & Dendrobium wardianum Warner. & May - Jun \\
\hline & 99 & Dendrobium wattii Rchb. f. & Dec - Jan \\
\hline & 100 & Dendrobium williamsonii Day \& Rchb.f. & Apr - May \\
\hline Dienia & 101 & Dienia ophrydis (J.König) Seidenf. & Jul - Aug \\
\hline \multirow[t]{3}{*}{ Epigeneium } & 102 & Epigeneium amplum (Lindl.) Summer. & Oct - Nov \\
\hline & 103 & Epigeneium fuscescens (Griff.) Summer. & Sep - Oct \\
\hline & 104 & Epigeneium rotundatum (Lindl.) Summer. & Mar-Apr \\
\hline Epipogium & 105 & Epipogium roseum (D.Don) Lindl. & May - Jun \\
\hline \multirow[t]{3}{*}{ Eria } & 106 & Eria coronaria (Lindl.) Rchb.f. & May - Jun \\
\hline & 107 & Eria spicata (D.Don) Hand.-Mazz. & Jul - Aug \\
\hline & 108 & Eria vittata Lindl. & Feb-Mar \\
\hline Eriodes & 109 & Eriodes barbata (Lindl.) Rolfe & Oct - Nov \\
\hline Erythrodes & 110 & Erythrodes blumei (Lindl.) Schltr. & Mar - Apr \\
\hline Esmeralda & 111 & Esmeralda Clarkei Rchb.f. & Dec - Feb \\
\hline Eulophia & 112 & Eulophia zollengeri (Rchb.f.) J.J. Smith & May - Jun \\
\hline Galeola & 113 & Galeola lindleyana (Hook.f. \& Thomson) Rchb.f. & May -Jun \\
\hline \multirow[t]{4}{*}{ Gastrochilus } & 114 & Gastrochilus calceolaris (Buch.-Ham. ex Sm.) D. Don & Mar-Apr \\
\hline & 115 & Gastrochilus distichus (Lindl.) Kuntze & May - Aug \\
\hline & 116 & Gastrochilusinconspicuus (Wall. ex Hook.f.) Kuntze & Jun-Jul \\
\hline & 117 & Gastrochilus pseudodistichus (King \& Pantl.) Schltr. & Aug - Oct \\
\hline \multirow[t]{2}{*}{ Goodyera } & 118 & Goodyera schlechtendaliana Rchb.f. & Aug-Sep \\
\hline & 119 & Goodyera viridiflora (Blume.) Lindl.ex Dietrich. & Sep \\
\hline \multirow[t]{2}{*}{ Habenaria } & 120 & Habenaria arietina Hook.f. & Aug-Sep \\
\hline & 121 & Habenaria dentata (Sw.) Schlter. & Sep-Oct \\
\hline Hygrochilus & 122 & Hygrochilus parishii (Veitch. \& Reichb.f.) Pfitzer & May - Jun \\
\hline \multirow[t]{7}{*}{ Liparis } & 123 & Liparis bistriata E.C. Parish \& Rchb.f. & Jun-Jul \\
\hline & 124 & Liparis bootanensis Griff. & Aug-Sep \\
\hline & 125 & Liparis distans C.B. Clarke & Oct - Nov \\
\hline & 126 & Liparis elliptica Wight. & Nov - Dec \\
\hline & 127 & Liparis nervosa (Thunb.) Lindl. & Jun-Jul \\
\hline & 128 & Liparis resupinata Ridl. & Oct - Nov \\
\hline & 129 & Liparis viridiflora (Blume) Lindl. & Oct - Nov \\
\hline
\end{tabular}


210 Orchid diversity in three districts of Nagaland, India

\begin{tabular}{|c|c|c|c|}
\hline \multirow{2}{*}{$\begin{array}{l}\text { Name of Genera } \\
\text { Luisia }\end{array}$} & \multicolumn{2}{|r|}{ Name of Species } & \multirow{2}{*}{$\begin{array}{c}\begin{array}{c}\text { Flowering } \\
\text { Season }\end{array} \\
\text { Apr- May }\end{array}$} \\
\hline & 130 & Luisia trichorrhiza (Hook.) Blume & \\
\hline \multirow[t]{4}{*}{ Oberonia } & 131 & Oberonia acaulis Griff. & Oct \\
\hline & 132 & Oberonia mucronata (D.Don) Ormerod \& Seidenf. & Jun - Jul \\
\hline & 133 & Oberonia obcordata Lindl. & Aug-Sep \\
\hline & 134 & Oberonia pachyrachis Rchb.f. ex Hook.f. & Aug - Sep \\
\hline \multirow[t]{3}{*}{ Otochilus } & 135 & Otochilus albus Lindl. & May - Jul \\
\hline & 136 & Otochilus fuscus Lindl. & Nov - Dec \\
\hline & 137 & Otochilus lancilabius Seidenf. & Mar-Apr \\
\hline Panisea & 138 & Panisea tricallosa Rolfe. & Nov - Dec \\
\hline \multirow[t]{2}{*}{ Paphiopedilum } & 139 & Paphiopedilum hirsutissimum(Lind1.) Stein. & Apr - May \\
\hline & 140 & Paphiopedilum villosum var. boxallii (Rchb.f.) Pfitzer & Jan - Mar \\
\hline \multirow[t]{2}{*}{ Papilionanthe } & 141 & Papilionanthe teres(Roxb.) Schltr. & Feb - Mar \\
\hline & 142 & Papilionanthe vandarum (Rchb.f.) Garay & Feb - Mar \\
\hline Pecteilis & 143 & Pecteilis susannae (L.) Raf. & Aug-Sep \\
\hline Pendulorchis & 144 & $\begin{array}{l}\text { Pendulorchis himalaica (Deb, Sengupta \& Malick) Z.J. Liu, } \\
\text { Ke Wei Liu \& X.J. Xiao. }\end{array}$ & Nov - Dec \\
\hline \multirow[t]{2}{*}{ Phaius } & 145 & Phaius flavus (Blume) Lindl. & Mar-Apr \\
\hline & 146 & Phaius tankervilleae (Banks ex L'Her.) Blume & Apr - Jun \\
\hline \multirow[t]{4}{*}{ Phalaenopsis } & 147 & Phalaenopsis braceana (Hook.f.) Christenson & Apr - May \\
\hline & 148 & Phalanaeopsis difformis (Wall. ex Lindl.) Kocyan \& Schuit. & Jun - Jul \\
\hline & 149 & Phalaenopsis taenialis (Lindl.) E.A. Christ. \& Pradhan & May - Jun \\
\hline & 150 & Phalaenopsis yingjiangensis (Z.H.Tsi) Kocyan \& Schuit. & Jul - Aug \\
\hline \multirow[t]{3}{*}{ Pholidota } & 151 & Pholidota articulata Lindl. & Jun \\
\hline & 152 & Pholidota convallariae (E.C. Parish \& Rchb.f) Hook.f. & May - Jun \\
\hline & 153 & Pholidota pygmaea H.J. Chowdhery \& G.D. Pal & Nov - Dec \\
\hline \multirow[t]{6}{*}{ Pinalia } & 154 & Pinalia acervata (Lindl.) Kuntze & Jul-Aug \\
\hline & 155 & Pinalia amica (Rchb.f.) Kuntze & Mar - Apr \\
\hline & 156 & Pinalia excavata (Lindl.) Kuntze & Jun - Jul \\
\hline & 157 & Pinalia graminifolia (Lindl.) Kuntze & Jun - Jul \\
\hline & 158 & Pinalia paniculata (Lindl.) Kuntze & Mar - Apr \\
\hline & 159 & Pinalia pannea(Lindl.) Kuntze & May - Jul \\
\hline \multirow[t]{3}{*}{ Pleione } & 160 & Pleione humilis (Sm.) D. Don & Mar - Apr \\
\hline & 161 & Pleione maculata (Lindl.) Lindl. & Nov - Dec \\
\hline & 162 & Pleione praecox (Lindl.) D. Don & Sep - Nov \\
\hline Polystachya & 163 & Polystachya concreta (Jacq.) Garay \& Sweet & Jun - Jul \\
\hline Renanthera & 164 & Renanthera imschottiana Rolfe. & May - Jun \\
\hline Rhynchostylis & 165 & Rhynchostylis retusa Blume & May - Jun \\
\hline Schoenorchis & 166 & Schoenorchis gemmate (Lindl.) J.J. Sm. & May - Jun \\
\hline Smitinandia & 167 & Smitinandia micrantha (Lindl.) Holttum & Jun - Jul \\
\hline Spathoglottis & 168 & Spathoglottis pubescens Lindl. & Jun - Jul \\
\hline \multirow[t]{2}{*}{ Tainia } & 169 & Tainia latifolia Benth. ex Hook. & Mar - Apr \\
\hline & 170 & Tainia minor Hook.f. & Apr-May \\
\hline Thrixspermum & 171 & Thrixspermum laurisilvaticum (Fukuy.) Garay & May - Jun \\
\hline Thunia & 172 & Thunia alba (Lindl.) Rchb. f. & Jun - Aug \\
\hline Uncifera & 173 & Uncifera obtusifolia Lindl. & Aug - Sep \\
\hline \multirow[t]{6}{*}{ Vanda } & 174 & Vanda alpina (Lindl.) Lindl. & Jun - Aug \\
\hline & 175 & Vanda ampullaceaum (Roxb.) L.M.Gardiner & Mar - Apr \\
\hline & 176 & Vanda bicolor Griff. & Jan - Feb \\
\hline & 177 & Vanda coerulea Griff. ex Lindl. & Sep - Oct \\
\hline & 178 & Vanda pumila Hook.f. & Mar-Apr \\
\hline & 179 & Vanda testacea (Lindl. ) Rchb.f. & May - Jun \\
\hline Vandopsis & 180 & Vandopsis undulata (Lindl.) J.J.Sm. & Mar-Apr \\
\hline
\end{tabular}


Table 3.Orchid genera represented by number of species and its habitat

\begin{tabular}{|c|c|c|c|}
\hline $\begin{array}{l}\text { Sl. } \\
\text { No. }\end{array}$ & Name of Orchid Genera & $\begin{array}{c}\text { Number of } \\
\text { species }\end{array}$ & Type/ Habitat \\
\hline 1 & Acampe Lindl. & 3 & Epiphytic \\
\hline 2 & Acanthephippium Blume. & 1 & Terrestrial \\
\hline 3 & Aerides Lour. & 2 & Epiphytic \\
\hline 4 & Agrostophyllum Blume. & 1 & Epiphytic \\
\hline 5 & Anthogonium Wall. ex Lindl & 1 & Terrestrial \\
\hline 6 & Arachnis Blume. & 3 & Epiphytic \\
\hline 7 & Arundina Blume & 1 & Terrestrial \\
\hline 8 & Bulbophyllum Thou. & 17 & Epiphytic \\
\hline 9 & Bulleyia Schltr. & 1 & Epiphytic/ Lithophytic \\
\hline 10 & Calanthe R.Br. & 6 & Terrestrial \\
\hline 11 & Chiloschista Lindl. & 1 & Epiphytic \\
\hline 12 & Cleisocentron Bruhl & 1 & Epiphytic \\
\hline 13 & Cleisostoma Blume & 3 & Epiphytic \\
\hline 14 & Coelogyne Lindl. & 15 & Epiphytic/ Lithophytic \\
\hline 15 & Cremastra Lindl. & 1 & Terrestrial \\
\hline 16 & Crepidium Blume & 3 & Terrestrial \\
\hline 17 & Cryptochilus Wall. & 2 & Epiphytic/ Lithophytic \\
\hline 18 & Cymbidium Sw. & 9 & Epiphytic/Lithophytic \\
\hline 19 & Dendrobium Sw. & 29 & Epiphytic \\
\hline 20 & Dienia Lindl. & 1 & Terrestrial \\
\hline 21 & Epigenium Gagnep. & 3 & Epiphytic/ Lithophytic \\
\hline 22 & $\begin{array}{l}\text { Epipogium J.G. Gmelin ex } \\
\text { Borkhausen }\end{array}$ & 1 & Saprophyte \\
\hline 23 & Eria Lindl. & 3 & Epiphytic/ Lithophytic \\
\hline 24 & Eriodes Rolfe & 1 & Epiphytic/ Lithophytic \\
\hline 25 & Erythrodes Blume & 1 & Terrestrial \\
\hline 26 & Esmeralda Rchb.f. & 1 & Epiphytic \\
\hline 27 & Eulophia R.Br. ex Lindl. & 1 & Saprophyte \\
\hline 28 & Galeola Lour. & 1 & Terrestrial \\
\hline 29 & Gastrochilus D.Don & 4 & Epiphytic \\
\hline 30 & Goodyera $\mathrm{R} . \mathrm{Br}$. & 2 & Terrestrial \\
\hline 31 & Habenaria Willd. & 2 & Terrestrial \\
\hline 32 & Hygrochilus Pfitzer & 1 & Epiphytic \\
\hline 33 & Liparis Rich. & 7 & Epiphytic/ Lithophytic \\
\hline 34 & Luisia Gaud. & 1 & Epiphytic \\
\hline 35 & Oberonia Lindl. & 4 & Epiphytic \\
\hline 36 & Otochilus Lindl. & 3 & Epiphytic \\
\hline 37 & Panisea Lindl. & 1 & Epiphytic \\
\hline 38 & Paphiopedilum Pfitzer & 2 & Epiphytic/ Lithophytic \\
\hline 39 & Papilionanthe Schltr. & 2 & Epiphytic \\
\hline 40 & Pecteilis Rafin. & 1 & Terrestrial \\
\hline 41 & $\begin{array}{l}\text { Pendulorchis Z.J. Liu, Ke Wei Liu } \\
\text { \& G.Q. Zhang }\end{array}$ & 1 & Epiphytic \\
\hline 42 & Phaius Lour. & 2 & Terrestrial \\
\hline 43 & Phalaenopsis Blume & 4 & Epiphytic \\
\hline 44 & Pholidota Lindl. ex Hook. & 3 & Epiphytic \\
\hline 45 & Pinalia Lindl. & 6 & Epiphytic \\
\hline 46 & Pleione D.Don & 3 & Epiphytic/ Lithophytic \\
\hline 47 & Polystachya Hook. & 1 & Epiphytic \\
\hline 48 & Renanthera Lour. & 1 & Epiphytic \\
\hline 49 & Rhynchostylis Blume & 1 & Epiphytic \\
\hline 50 & Schoenorchis Blume & 1 & Epiphytic \\
\hline
\end{tabular}


212 Orchid diversity in three districts of Nagaland, India

\begin{tabular}{|l|l|c|l|}
\hline $\begin{array}{l}\text { Sl. } \\
\text { No. }\end{array}$ & \multicolumn{1}{|c|}{ Name of Orchid Genera } & $\begin{array}{c}\text { Number of } \\
\text { species }\end{array}$ & \multicolumn{1}{|c|}{ Type/ Habitat } \\
\hline 51 & Smitinandia Holtt. & 1 & Epiphytic \\
\hline 52 & Spathoglottis Blume & 1 & Terrestrial \\
\hline 53 & Taenia Blume & 2 & Terrestrial \\
\hline 54 & Thrixspermum Lour. & 1 & Epiphytic \\
\hline 55 & Thunia Rchb.f. & 1 & Epiphytic \\
\hline 56 & Uncifera Lindl. & 1 & Epiphytic \\
\hline 57 & Vanda W.Jones ex R. Br. & 6 & Epiphytic \\
\hline 58 & Vandopsis Pfitzer & 1 & Epiphytic/ Lithophytic \\
\hline
\end{tabular}

[Epiphytic: 30; Terrestrial: 15; Mixed (Terrestrial/Epiphytic): 11; Saprophyte: 2]

Crepidium acuminatum, C. calophyllum, C. khasianum, Dienia ophrydis, Eriodes barbata, Erythrodes blumei, Goodyera viridiflora, Habenaria arietina, Habenaria dentate,Pecteilis susannae, Phaius tankervilleae, Tainia latifolia.

Temperate vegetation: During the present study it was observed that majority of the rare species were found in this vegetation type. Epiphytic orchid species viz., Acampe rigida, A. multiflora, Agrostophyllum callosum, Arachnis senapatiana, Bulbophyllum affine, B.candidum, B. crassipes, B. cylindraceum, B. elatum, B. helenae, $B$. odorotissimum, $B$. reptans, B. retusiusculum, $B$. sunipia, $B$. umbellatum, $B$. viridiflorum, Bulleyia yunnanensis, Coelogyne barbata, C. calcicola, C. corymbosa, C. cristata, C. griffithii, C. nitida, C. occultata, C. stricta, Cryptochilus luteus, C. sanguineus, Cymbidium cochleare, $C$. devonianum, $C$. elegans, $C$. erythraeum, $C$. mastersii, C. tigrinum, Dendrobium aphyllum, D. chrysanthum, D. crepidatum, D. densiflorum, D. denudans, D. devonianum, D. ochreatum, D. falconeri, $D$. fimbriatum, D. heterocarpum, D. lituiflorum, D. longicornu, D. nobile, D. sociale, D. wardianum, D. wattii, D. williamsonii, Epigeneium fuscescens, E. rotundatum, Eria coronaria, E. spicata, Esmeralda clarkei, Gastrochilus calceolaris, G. distichus, G. pseudodistichus, Hygrochilus parishii, Liparis bistriata, L. resupinata, Oberonia acaulis, O. mucronata, O. obcordata, O. pachyrachis, Otochilus albus, O. fuscus, O. lancilabius, Panisea tricallosa, Paphiopedilum hirsutissimum, P. villosum var. boxallii, Papilionanthe vandarum, Pendulorchis himalaica, Phalaenopsis braceana, P. taenialis, P. yingjiangensis, Pholidota articulata, $P$. convallariae, Pinalia excavata, $P$. graminifolia, $P$. paniculata, $P$. pannea, Pleione humilis, P. praecox, Renanthera imschottiana, Schoenorchis gemmata, Thrixspermum laurisilvaticum, Thunia alba, Vanda alpina, V. pumila, Vandopsis undulate. Terrestrial orchids are Calanthe alismifolia, C. puberula, Cremastra appendiculata, Epipogium roseum, Eulophia zollengeri, Galeola lindleyana, Goodyera schlechtendaliana, Phaius flavus.

Sub Alpine/Alpine vegetation: This type of forest occurs only in high altitudinal area in Saramati range of Kiphire district and is predominated with Rhododendron species (Particulary Rhododendron macabeanum and Rhododendron arboreum). Species of Epigenium, Pleione and Goodyera are some rare species of the region.

The state has witnessed a large scale exploitation of orchid species in the past owing to their horticultural value, moreover the practice of logging, shifting cultivation and unplanned developmental activities which has resulted in decrease in plant biodiversity of the region including orchids as being vulnerable to changes in microclimate. 
Rao et al. (2003) listed 105 species under 46 genera of the family Orchidaceae from India in different threat categories out of which 14 species are reported from these three districts and these areEsmeralda clarkei (Arachnis clarkei), Bulbophyllum rotschialdianum, Bulleyia yunnanensis, Calanthe alismaefolia, Coelogyne barbata, Coelogyne cristata, coelogyne flaccida, Coelogyne nitida, Coelogyne prolifera, Galeola lindleyana, Paphiopedilum hirsutissimum, Renanthera imschottiana, Vanda coerulea, Pholidota imbricate (Pholidota calceata).

Further in this study it is found that wild population of threatened species like Coelogyne barbata, Coelogyne cristata and Vanda coerulea are being collected by the people to adorn their houses causing further declining the population. Wild flowering population of Paphiopedilum villosum var boxallii from Pangsha village bordering Myanmar in Tuensang district, report of Arachnis labrosa var.zhaoi and Thrixspermum laurisilvaticum as new records for India are some of the interesting findings.

It should be noted that all orchid species are included under Appendix B of the Convention on International Trade in Endangered Species of Wild Fauna and Flora (CITES) and subsequently all Paphiopedilum species (Lady's slippers) are listed under Appendix I of CITES, hence a collaborative effort from the government agency and researchers alike could thus go a long way in conservation of the remaining orchid species.

Indian orchids are mostly confined to North-Eastern region and Western Ghats. Unfortunately, in recent years excessive human interventions have led to the rapid decline of floral diversity and as orchids are being intolerant to slight changes in microclimates causing massive decline of its population. Hence, the need for conservation of orchids both in-situ and ex-situ is necessary for further sustainable utilization of this rich resource.

\section{Acknowledgements}

Authors are thankful to Department of Biotechnology, Ministry of Science \& Technology, Govt. of India, New Delhi for financial support through a R\&D project to Prof. C. R. Deb vide order No. DBT/NER/Agri/23/2013. Facilities used from the Institutional Biotech Hub, UGC-SAP (DRS-III) programmes are duly acknowledged.

\section{LITERATURE CITED}

Atwood, J.T. 1986. The size of orchidaceae and the systematic distribution of epiphytic orchids. Selbyana 9(1): $171-186$.

Chankija, S.; Kumar, Y. \& Gurung, P.B. 1992. Orchids of Nagaland. Forest Department, Nagaland.

Clarke, C.B. 1889. On the plants of Kohima and Muneypore. J. Linn. Soc. 25: $71-74$.

Deb, C.R \& Imchen, T. 2008. Orchid Diversity of Nagaland. Sci Chem Publishing House, Udaipur, Rajasthan, India.

Deb, C.R. \& Imchen, T. 2011. Orchids of horticultural importance from Nagaland, India. Pleione 5(1): $44-48$.

Deb, C.R.; Deb, M.S.; Jamir, N.S. \& Imchen, T. 2009. Orchids in indigenous system of medicine in Nagaland, India. Pleione 3(2): 209 - 211.

Deb, C.R.; Jamir, N.S.; Jakha, H.Y.; Kikon, Z.P. \& Dey, S. 2014. Esmeralda clarkei Reichenbach f. (Orchidaceae): a new report for the flora of Nagaland, India. Pleione $8(1): 181-183$. 
214 Orchid diversity in three districts of Nagaland, India

Deb, C.R.; Jakha, H.Y. \& Dey, S. 2015. Report of Dendrobium regium Prain (Orchidaceae) from Nagaland, India - a new record for North-East India. Pleione 9(1): 258 - 260.

Deb, C.R.; Jakha, H.Y. \& Jamir, N.S. 2016. Polystachya concreta (Jacquin) Garay \& H.R. Sweet (Orchidaceae): a new record for Nagaland, India. Pleione 10(1): $189-$ 191.

Deb, C.R.; Kamba, J.; Longchar, T.B. \& Jakha, H.Y. 2017. Cymbidium bicolor Lindl. (orchidaceae): a new report for the orchid flora of Nagaland, India. Pleione 11(2): $498-500$.

Deorani, S.C. \& Naithani, H.B. 1995. Orchids of Nagaland. Oriental Enterprises, Dehradun, India.

Dressler, R.L. 1993. Phylogeny and classification of the orchid family. Miss. Bot. Card. Cambridge University 47: 25 - 67.

Haywood, W.H. 1993. Flowering Plants of the World. B. T. Batsford Ltd., Oxford.

Hooker, J.D. 1890. Orchidaceae. In: The Flora of British India. 5: 667 - 858.1888; 6:1 198. Ashford, Kent. London.

Hynniewta, T.M.; Kataki, S.K \& Wadhwa, B.M. 2000. Orchids of Nagaland. Botanical Survey of India, Calcutta.

Imchen, T.;Jakha, H.Y. \& Deb, C.R. 2015. Phalaenopsis braceana (Hooker $f$.) Christenson - a new distributional record for Nagaland, India. Pleione 9(1): 244 $-246$.

Jain, S.K. \& Rao, R.R. 1977. A Handbook of Field and Herbarium Methods. Today and Tomorrow's Printers and Publishers, New Delhi.

Jakha, H.Y. \& Dey, S. 2017. Addition of Crepidium calophyllum (Rchb.f.) Szlach.,Eriodes barbata (Lindl.) Rolfe and Erythrodes blumei (Lindl.) Schltr. to the orchid flora of Nagaland, India. Indian J. Fundam. Appl. Life Sci. 7(2): 1 - 4.

Jakha, H.Y.; Deb, C.R.; Dey, S. \& Jamir, N.S. 2014a. Arachnis senapatiana (Phukan \& A.A. Mao) Kocyan \& Schuiteman (Orchidaceae): an addition to the flora of Nagaland, India. Pleione 8(2): $516-518$.

Jakha, H.Y.; Deb, C.R.; Dey, S.; Jamir, N.S. \& Kikon, Z.P. 2014b. Dendrobium tamenglongense R. Kishor; Y.N. Devi; H.B. Sharma; J. Tongbram \& S.P. Vij: Orchidaceae: an addition to the flora of Nagaland. Pleione 8(2): $526-528$.

Jakha, H.Y.; Deb, C.R.; Singh, S.K.; Verma D.; Dey, S. \& Jamir, N.S. 2015 a. Pendulorchis (Orchidaceae) - A new generic record for Nagaland, India. Keanean J. Sci. 4: 31-34.

Jakha, H.Y.; Deb, C.R.; Dey, S. \& Jamir, N.S. 2015b. Arachnis labrosa var. zhaoi (Orchidaceae): A new record for India. Rheedea 25(2): 120 - 122.

Jamir, A.; Jamir, N.S. \& Deb, C.R. 2015a. Three New Distributional Records of Orchid from Intangki National Park, Nagaland, India. Int. J. Res. Studies Biosci. 3(6): 7 - 9.

Lawler, L.J. \& Rao, A.N. 2002. Medicinal uses of orchids in some Asian countries. J. Trop. Med. Pl.3(1): $73-88$.

Mabberley, D.J. 1998. The Plant Book ( $2^{\text {nd }}$ Edn.). Cambridge University Press, Cambridge. Madras.

Odyuo, N.; Deori, C. \& Daimary, R. 2016a. Panisea panchaseensis (Orchidaceae): an addition to the flora of India. Richardiana 16: $141-145$. 
Odyuo, N.; Deori, C. \& Daimary, R. 2016b. Coelogyne calcicola (Orchidaceae): an addition to the flora of India. Richardiana 16: 343-346.

Odyuo, N.; Deori, C. \& Daimary, R. 2017. Dendrobium tuensangense, a new species of Orchidaceae from Nagaland, Northeast India. Phytotaxa 311(2): 185-189.

Rao., C.K.; Geetha., B.L. \& Geetha., S. 2003. Red List of Threatened Vascular Plant Species in India. Compiled from the 1997 IUCN red list of threatened plants. ENVIS, Botanical Survey of India Ministry of environment \& forests. Dehradun.

WCSP. 2017. Kew Royal Botanic Gardens. World Checklist of Selected Plant Families. URL: http://apps.kew.org/wcsp/home.do (Accessed: May, 2017). 